\title{
Effect of the thermoforming process variables on the sheet friction coefficient
}

\author{
Rosa A. Morales a , María V. Candal ${ }^{\mathrm{a}, *}$, Orlando O. Santana ${ }^{\mathrm{b}}$, Antonio Gordillo ${ }^{\mathrm{b}}$, Rubén Salazar ${ }^{\mathrm{a}, 1}$ \\ ${ }^{a}$ Grupo de Polímeros USB, Departamento de Mecánica, Universidad Simón Bolívar, Apartado 89000, Caracas 1080-A, Venezuela \\ b Centre Català del Plàstic, Universitat Politècnica de Catalunya, c/Colom 114, 08222 Terrassa, Barcelona, Spain
}

\section{A R T I C L E I N F O}

\section{Article history:}

Received 26 October 2012

Accepted 4 August 2013

Available online 14 August 2013

\section{Keywords:}

Thermoforming

Material testing

Friction coefficient

Polypropylene

\begin{abstract}
A B S T R A C T
The objective of this work was to obtain, through a simple experimental assembly, the real influence of the friction and process conditions in the assisted thermoforming process. The use of simulation tools to optimize this process requires the knowledge of the sheet-plug system friction coefficient [ $\mu]$, the used temperature and velocity conditions, and due to that, the software does not have a wide database. For this, the users suppose $\mu$ with the previous known difference between the simulated and the experimental data. For this reason, the obtaining of $\mu$ may allow to achieve more accurate simulations. In this work, a modified pendulous impact in Izod geometry was used to the measurements. The $\mu$ increased with the sheet temperature. On the other hand, it was not possible to detect a significant $\mu$ variations regarded with the plug material.
\end{abstract}

(c) 2013 Elsevier Ltd. All rights reserved.

\section{Introduction}

Although thermoforming is one of the less used techniques to produce plastic objects, nowadays it is the higher growing process in the industry due to its economical advantages and the improvement of the process variables. All this has allowed reaching a wide range of pieces with a better quality $[1,2]$.

The process begins with a prefabricated piece, which in most of the cases it is a film or a sheet, in which it is applied a limited hot quantity, able to make it highly flexible and elastic, but maintaining enough dimensional flexibility to avoid a deformation caused by gravity. At this point, relative low forces are required to make the sheet which adopts the mold's shape. After that, the piece shall be cooled to recover its strength, and finally, the excess of material on the edges shall be cut [3].

The thermoforming is an extremely difficult process to analyze. It involves a three-dimensional state of deformities in a viscoelastic body with multiple choices, regarding the conditions of the process [4-6] and very complex geometrics [7,8]. Moreover, each step of the process shall be particularly analyzed and only the forming steps may have multiple groups of involved variables, depending on the specific used technique. Despite all these difficulties, the benefits of understanding and predicting in which way may be affected the final properties of a thermoformed piece are obvious, when some specific parameters in the molding are altered [9].

\footnotetext{
* Corresponding author. Tel.: +58 212 9064065; fax: +58 2129064062.

E-mail address: mcandal@usb.ve (M.V. Candal).

1 Deceased.
}

In previous works, the influence of the different variables involved in the thermoforming process was studied. These variables are the following: sheet temperature [10-13], plug rate [12], plug material $[13,14]$, plug temperature [13-16], surface finish [14$16]$, plug geometry [11,12], and air pressure [12]. It is difficult to isolate a specific parameter to study its effect, because in most of the cases, a single parameter may alter another one. Thus, for example, an increase in the initial temperature of the sheet may decrease its viscosity and alter its friction properties.

\section{Friction and friction coefficient}

Since the forming process may imply mechanic interaction between the softened sheet and the mold and/or plug material, the friction phenomenon between these elements has an important role in the quality of the molded pieces. This may be clearly observed in the forming techniques by dragging and forming assisted by piston techniques.

The main influence of the friction is based on determining if an adherence or slide shall be produced at the moment that the sheet makes contact with the mold or the plug. If the sheet sticks to the mold, which is very common when there is a high friction, the sheet shall not continue suffering stretching and shall keep its thickness. By other hand, if the friction is low, the stretching of the sheet on the mold shall permit its deformation and that means a thickness decrease in the lower sections of the piece [10].

What is tried to achieve in the assisted forming processes is a high friction between the plug and the sheet, because the main objective of the process is to compensate the low thickness 
obtained in the deepest pieces formed in negative or female molds. In this way, a more uniform thickness distribution may be achieved. Nevertheless, in the dragging forming, the friction shall be controlled to avoid excessively thin upper sections.

The friction is governed by parameters that depend on the design, the involved materials, and the processing conditions. Illig [1] pointed out three factors that were found not only in the sheet, but also in the forming tool. These factors defined the magnitude of the friction force: fabrication material (sheet and plug), temperature (sheet and plug), and superficial treatment (roughness) [17].

The friction force $f$ is defined as follows:

$f=\mu N$

where $N$ represents the normal force acting in the interface between two materials and $\mu$ is an adimensional parameter known as friction coefficient. Between these two parameters, $\mu$ is the most characteristic parameter, since $N$ depends on the conditions of the charge system. Moreover, in a previous work, Gascó et al. [10] concluded that, through measurements of $\mu$ under different charge conditions, $\mu$ did not experience important changes with $N$. In a later study, Laroche et al. [15] evidenced that $\mu$ has a dependency with the temperature and its influence on the distribution of the thickness.

$\mu$ is a measure of the opposition by a two-bodies-system to the slide between them. There are two types of identified coefficients: a static one $\left(\mu_{s}\right)$, related to the resistance made by the system at the beginning of the movements, and a dynamic or kinetic $\left(\mu_{d}\right)$, which is presented once the movement has begun. $\mu$ is bigger than $\mu_{d}$, and this one depend on the velocity in which the slide is produced.

Normally, the $\mu$ measurements are made by quantifying the force $f$ that exists among the samples of known weight sliding between them in a constant velocity and then calculating $\mu$ through Eq. (1). There are several experimental assemblies that may be made for this purpose, and many of them have been normalized for the American Society for Testing and Materials (ASTM).

A typical $\mu$ measurement test it is shown in Fig. 1. In a variable inclination diagram $(\phi)$, which its surface is covered by one of the testing materials, it has been set a sliding block made with the other testing material of $m$ mass. The normal force exerted on the block is determined by $m \cos \phi$, while the friction force that acts in the movement diagram is determined by $m \sin \phi$. Eq. (2) consists in:

$\mu=\frac{f}{N}=\frac{m g \sin \varphi}{m g \cos \varphi}=\tan \varphi$

If the diagram's inclination is increased until the movement of the block begins, the value of $\phi$ allows obtaining $\mu$. According to the aforementioned, once the movement is initiated, this will be able to continue to a less $\phi$. Since the block falls due to the gravity, it has a variable velocity, and that is the reason why this assembling is not suitable for the $\mu_{d}$ measure.

The ASTM: D3028 and the ASTM: D1894 norms also describe procedures to determine $\mu$ between plastic materials or between plastics and other kind of materials.

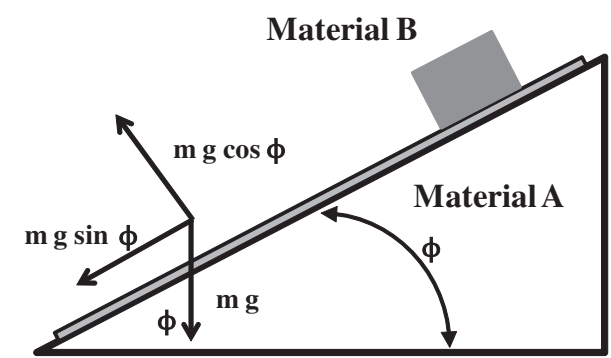

Fig. 1. Experimental assembling for the measurement of the static friction coefficient $\left(\mu_{s}\right)[9]$.

\section{Previous works}

There are other experimental studies about the influence of the friction coefficient in assisted thermoforming [11,13-16]. To be aware of the importance of $\mu$ in this process has also encouraged the study of techniques different to the described processes in the ASTM norms to determine the friction coefficient and its dependency on variables such as the temperature and roughness, in the velocity ranges that are very common in the forming processes.

Collins et al. [14] made a study of the influence of plug-sheet slide in the thickness distribution of molded pieces by assisted forming and they used a process modification of the ASTM: D1894 norm that uses a "sleight" that slides with constant velocity on a plastic sheet using a dynamometer to measure the needed force to achieve such condition.

Additionally, Gascó et al. [10] compiled different assembling to measure $\mu$, and some of them are based on common measurement equipments in polymer engineering, like the universal testing and measurement of high impact machines. Although such study was focus on the interaction between same material polymer surfaces, the used assembling may be adapted to similar measures between polymers and other kind of materials (metals, wood, etc.). By other hand, Laroche et al. [11] used a torsional rheometer to measure both $\mu_{d}$ and $\mu_{s}$, considering that it is a more complex and expensive device than aforementioned devices.

In a later study, Hegemann and Eyere [16] developed a new technique to measure $\mu$ in a wide temperature and velocity range, in process conditions similar to those used during the assisted thermoforming. They observed a $\mu$ dependency with the temperature in HDPE and HIPS sheets, when using steel assistant and thermoplastic foam (HYTAC ${ }^{\circledR}$ BIX). There were not found significant differences in the velocity at low temperatures, while at high temperatures a light increase in $\mu$ was observed. Additionally, the results of the simulation showed a strong influence of $\mu$ in the force exerted by the needed plug to deform the sheet.

Tulsian et al. [13] researched the effect of plug material (synthetic and non-synthetic thermoplastic foam, synthetic epoxy) and the temperature of the sheet on the PṔs $\mu$, using a simulation commercial program of the thermoforming process. These authors varied in the simulator program the $\mu$ value, until they found that the predicted thickness distribution values were similar to the thickness obtained experimentally.

\section{Experimental details}

It was decided to use the suggested method by Gascó et al. [10], making a minor modification to the pendulous impact in Izod geometry, which allows to measure the $\mu$ in the velocity range in which it is made the forming in this study (between 0.7 and $3.0 \mathrm{~m} / \mathrm{s}$ ). Its working is explained in the following section. Fig. 2(a) shows a schematic representation of the experimental assembly. The pendulous impact equipment was modified by the addition of two components: a mobile device that was used to absorbed the hammer's energy during the impact and displaced the metallic sample adherent in its lower area, and a plate used as a support to the polymeric sheet and on which the mobile device displaced (Fig. 2).

\subsection{Measurement principle}

The pendulous impact equipment allows measuring the potential energy associated with a calibrated pendulum. This energy may be measured not only before, but also after the hammer interacts with any other element. Originally, the device is designed to measure the resistance to the impact of the plastic samples 


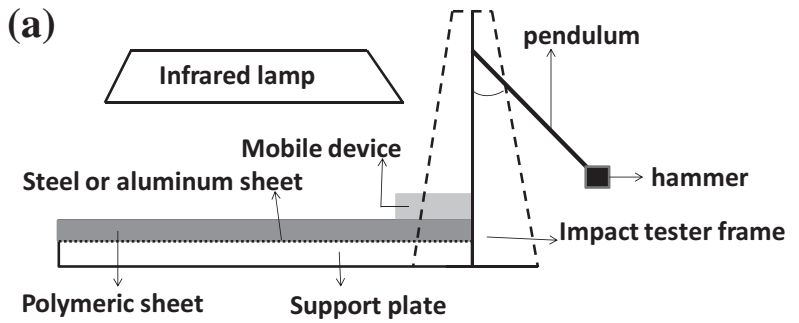

(b)

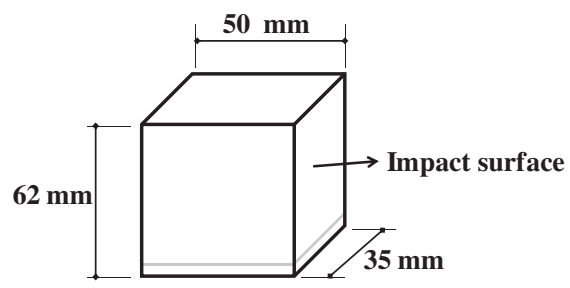

Fig. 2. Schematic representation of the experimental assembly design for the friction coefficient measurement.

through the procedure of the ASTM: D256 norm. In this case, the pendulum interaction with the sample originates its breaks and the lost of the pendulum's energy.

The pendulum's energy depends on two fundamental factors: the equivalent mass and the initial angular position. The measurement scale used in the equipment was in a range of $\theta$ from $-90^{\circ}$ to the $70^{\circ}$, for this study. This way, the initial potential energy $\left(E_{0}\right)$ may be calculated with the following equation:

$E_{0}=\frac{1}{2} U_{\text {MAX }}\left(1+\sin \theta_{0}\right)$

where UMAX represents the maximum energy value that a hammer may have (when the energy is in $\theta=-90^{\circ}$ ) and $\theta_{0}$ is the initial angle in which is set the hammer at the moment the measurement takes place.

The pendulum initial position is maintaining fix in $\theta=70^{\circ}$. In that position, the pendulums are calibrated to have an $E_{0}$ initial energy of $0.5,1,2$ or $4 \mathrm{~J}$. However, how it is showed Eq. (3), the initial energy may be varied modifying $\theta_{0}$ value and this way a wide range may be covered, either of energy or initial velocity of the pendulum.

This $E_{0}$ shall be totally transformed in kinetic energy in $\theta=-90^{\circ}$ angular position, which is where it is the pendulous interaction created with the other body, assuming the energy conservation and not considering he loss of friction with the air, in the impact-device contact or test (type Brinnell indentation, among others).

In the friction measurements, and different from the measured resistance currents to the impact, the pendulum's interaction is not with a plastic sample to break, but with a mobile device to displace (Fig. 3). If the impact is approximately elastic, there may be energy transfer from the pendulum to the mobile device during the impact, thus:

$K_{0, C}=E-E_{0}$

where $K_{0, C}$ represents the initial kinetic mobile device's energy, and $E$ is the final potential energy of the pendulum. The mobile device kinetic energy may be related to the friction energy, through the definition of a non-conservative work of force $\left(W_{f}\right)$ :

$W_{f}=f d=K_{0, C}-K_{c}=K_{0, C}$

where $d$ is the displaced distance of the mobile device before stop and $K_{c}$ is the final kinetic energy of the mobile device that is equal to zero, because the mobile device stopped.

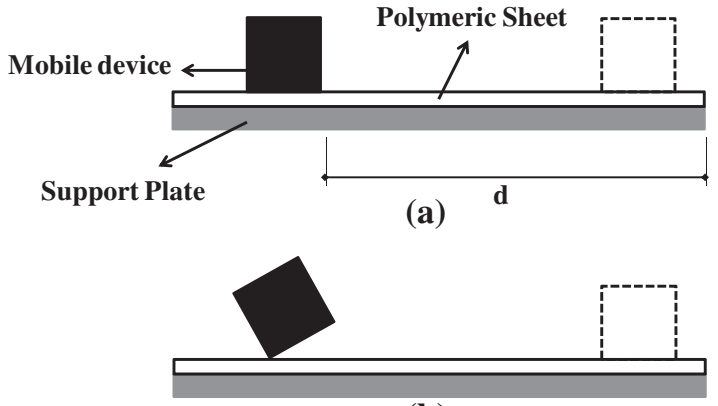

(b)

Fig. 3. Schematic representation of the displacement measurement (d) of the mobile device: (a) valid measurement and (b) discarded measurement.

The definition of friction force $f$ showed in Eq. (1) is substituted in Eq. (5) to obtain a definition of the friction coefficient $\mu$ according to the variables that may be experimentally measured: the initial energy (calculated from the initial angular position and the energy of the calibrated hammer), the final energy (read in the scale of the equipment), the $d$ mobile device displace, the mobile device's equivalent mass $m_{m}$; moreover, the acceleration of the gravity $g$ and $N$ acting in the interface between the materials (Eq. (6)). From the two showed parameters in Eq. (1), $\mu$ is the most characteristic because $N$ depend on the charge conditions of the system.

$\mu=\frac{E_{0}-E}{N d}=\frac{E_{0}-E}{m_{m} g d}$

\subsection{Test methodology}

The sheets were extruded using High-Impact Polystyrene HIPS Estizulia PS 4320 and Polypropylene PP Propilven F 409, with a melt flow index (MFI) of 8.5 and $4.0 \mathrm{~g} / 10 \mathrm{~min}$, respectively. Since friction is a clearly superficial phenomenon, the sheet thickness is not relevant for the tests.

The sheets of polymeric material were placed on a MDF support plate, using glue made of epoxic resin of fast cured. This material was chosen since the high temperatures dissipated less the heat provided to the polymeric sheet, and it allows the mobile device to move faster. The used commercial metallic material samples were the following: 1020 FERRUM Steel (C\% 0.15-0.23 max, Si\% $0.1-0.2, \mathrm{Mn} \%$ 0.30-0.60) and an aluminum 3003 alloy, fabricate by CVG-ALCASA (Si\% 0.20, Fe\% 0.65, Cu\% 0.128\%, Mn\% 1.042, Mg\% 0.003, Cr\% 0.00007, Ni\% 0.0028, Zn\% 0.0075, Ti\% 0.013, B\% 0.0022, $\mathrm{Pb} \% 0.0033$ were $35 \times 62 \mathrm{~mm}$ plates, which adhere to the lower part of the mobile device with a total mass of $100 \mathrm{~g}$ with $50 \times 62 \times 35 \mathrm{~mm}$ dimensions, as showed in Fig. 2(b). The samples, for both aluminum and steel samples, were subjected to tests in the sandpaper's bank to achieve variations in its roughness. 75 GRIT and 360 GRIT sandpapers were used.

Although, at first, sample with a finest finishing were used, these samples lost its finishing very fast, when the measurement were made; thus, they were discarded. An infrared lamp was used as heating system for the sheet.

It was made a calibration curb of the sheet temperature according to the high $\mathrm{h}$ of the infrared lamp, in order to find out the testing temperatures. Infrared thermometer was used taking care that variations were not higher than $\pm 5^{\circ} \mathrm{C}$.

The hammer was raised until a $\theta_{0}$ and it was freely dropped. In this case, $E$ and $d$ values were registered. The $d$ values were only considered valid if the mobile device did not rotate in its displacement (Fig. 3(a)). The measurements were discarded when the 
mobile device rotation was more than $20^{\circ}$ according to the support plate plane of the polymeric sheet (Fig. 3(b)).

\section{Results and discussion}

\subsection{Important aspects to consider $\mu$ measurement}

Since the assisted thermoforming process involves a mobile plug, the relevant friction coefficient is $\mu_{d}$. The main source of mistakes associated with the measurements is the assumption of elasticity from the collision between the mobile device and the hammer. The previous measurements produced unexpected results. For example, $\mu_{d}$ to 1 , even for systems where were reported values around 0.4 (value of the simulation software). The analysis of Eq. (6) showed that from the five involved variables, the one related to the initial energy of the mobile device (initially assumed as $E_{0}-E$ ) was less precisely measured.

It is known at the present that the collision is inelastic; thus, a total recuperation shall be not assumed. In other words, all the materials evidenced certain degree of "non-conservative state." Therefore, the inelasticity phenomenon shall be included in its experimental analysis. For this reason, the initial kinetic energy of the mobile device was overvalued, because there were considerable energy losses during the collision $[18,19]$.

To study this effect, a restitution coefficient concept $(\varepsilon)$ was used as an empiric parameter to study the loss of energy during the impact, and consequently, this concept permitted the inelasticity achieved by the material during an impact test. It is defined as the relation between the applied velocity before the collision $(v 0)$ and the velocity after the collision $(v 1)$. This coefficient always has a positive value and its limits are one for the purely elastic contact and zero for an inelastic one [20].

This $\varepsilon$ may be calculated by two ways: The $v_{0}$ value may measured by an impact test by fall or it may be directly measured using a photoelectric cell.

Based on the aforementioned, there were two modifications tried to make the equipment in order to measure the real energy of the mobile device at the initial moment of the movement. First, it was considered to use a photoelectric cell set just in the initial point of the movement that allowed determining the time that takes the mobile device to move through the cell light beam. With this time, and the mobile device longitude may be calculated the initial velocity, and it may be applied the kinetic energy definition to establish the kinetic energy at the beginning of the movement. The mobile device mass value was also known. Sadly, the reduce space in the experimental assembling made impossible to set the cell in the desired point, and this method was discarded.

Sanchez-soto et al. [18] and Martínez et al. [19] in a previous work suggested alternative methods to calculate $\varepsilon$ using the collision impact technique by fall in order to quantify the impact energy loss between the two materials incorporating $\varepsilon$.

A non-conservative serial model and a non-lineal (spring/shock absorber) were suggested to reproduce the material behavior. The model considered both the identification phenomenon and the flexion that takes place during the impact through the restitution coefficient.

Additionally, due to Sanchez-Soto et al. [18] have found the $\varepsilon$ variation with the velocity, then it was tried to use an ultrasound sensor, set in a way that the sound wave hit frontally the mobile device while it was moving. With this assembly, it is possible to determine the velocity and even the acceleration according to the mobile device movement. Having these data, it can be designed a coefficient profile, $\mu$ according to velocity for each temperature and roughness condition. Nevertheless, the only available equipment was not able to register the measurements with the requested velocity. It was only possible to make a measurement each $0.5 \mathrm{~s}$, period of time even longer than the phenomenon in some cases.

For this reason, the $\mu_{d}$ measured values in this study were always higher than those reported by the commercial simulation programs for the aluminum-plastic and steel-plastic interaction. However, the program permits to establish comparisons regarding materials, velocities, and different temperatures.

Another less important cause of mistakes was that the movement of the mobile device was not always rectilinear. In occasions, some considerable rotations have been observed, which is produced by irregularities on the sheet. There were zones that originated more friction than others in which a side of the mobile device stopped before than the opposite side by braking, causing the rotation (Fig. 3(b)). However, the data reproduction was acceptable. Also, the precision of the measurements were acceptable having always less than five percent of uncertainty.

In other words, despite the limitations of the experimental assembly, the measurement precision evidenced may be considered as acceptable, since one of the most important parts for the measures is to exert an initial impact, and magnitude of which may be controlled. However, it may be justified the addition of another measurement velocity implement, like an ultrasound sensor able to make measures with enough speed (for example each $0.1 \mathrm{~s}$ ). By this way, it may be possible to calculate $\varepsilon$ to solve the problem of assembling.

\subsection{Effect of the process conditions}

The influence of the process conditions and the characteristics of the Plug about $\mu_{d}$ were evaluated. The four evaluated parameters were the following: material of the plug (aluminum and steel), temperature of the polymeric sheet, velocity of the mobile device displacement (equivalent to the velocity of plug's penetration), and superficial finishing of the plug.

\subsubsection{Effect of the sheet temperature}

Fig. 4 shows the variation of $\mu_{d}$ regarding the temperature of the sheet for aluminum and steel samples with superficial finishing achieved with the help of GRIT 75 sanding, displacing with an initial velocity of $1.6 \mathrm{~m} / \mathrm{s}$ and $2.1 \mathrm{~m} / \mathrm{s}$, Fig. 4(a) and (b), respectively. In these figures is evidenced the increase in $\mu$ with the temperature in a tendency that looks like an apparently asymptotic behavior. The increase in $\mu_{d}$ with the temperature has been verified for other commons plug-polymer systems [19,20], although there is no clear reason for this behavior. The limited number of points is due to a less control of the heating device (infrared lamp).

The most important fact observed when the temperature changed was that for the values higher than $115^{\circ} \mathrm{C}$, it was not possible to continue the measurements, because the mobile device overturned when passing on the hot zone, which is considered an evidence of a metal-polymer adherence phenomenon also observed in a previous study for systems, PP-foam polymers, and PP aluminum [21]. In other words, for temperatures of the sheet in the typical range of HIPS thermoforming, the sheet adheres to the plug; thus, $\mu_{d}$ reaches values higher than 1 .

Nevertheless, it is important to mention that this effect was verify under used experimental conditions (with the metallic material at room temperature) that is the reason why it is not possible to make explorations for the case of real manufacturing conditions, in which molds heated high above the room temperatures, to the point that sometimes they need cooling systems. Unfortunately, it was not possible to have the necessary resources to reach an optimal metal heating, in order to verify if the adherence was possible under such temperature conditions; nevertheless, the aforementioned study [22,23] reported this condition. On that case, 

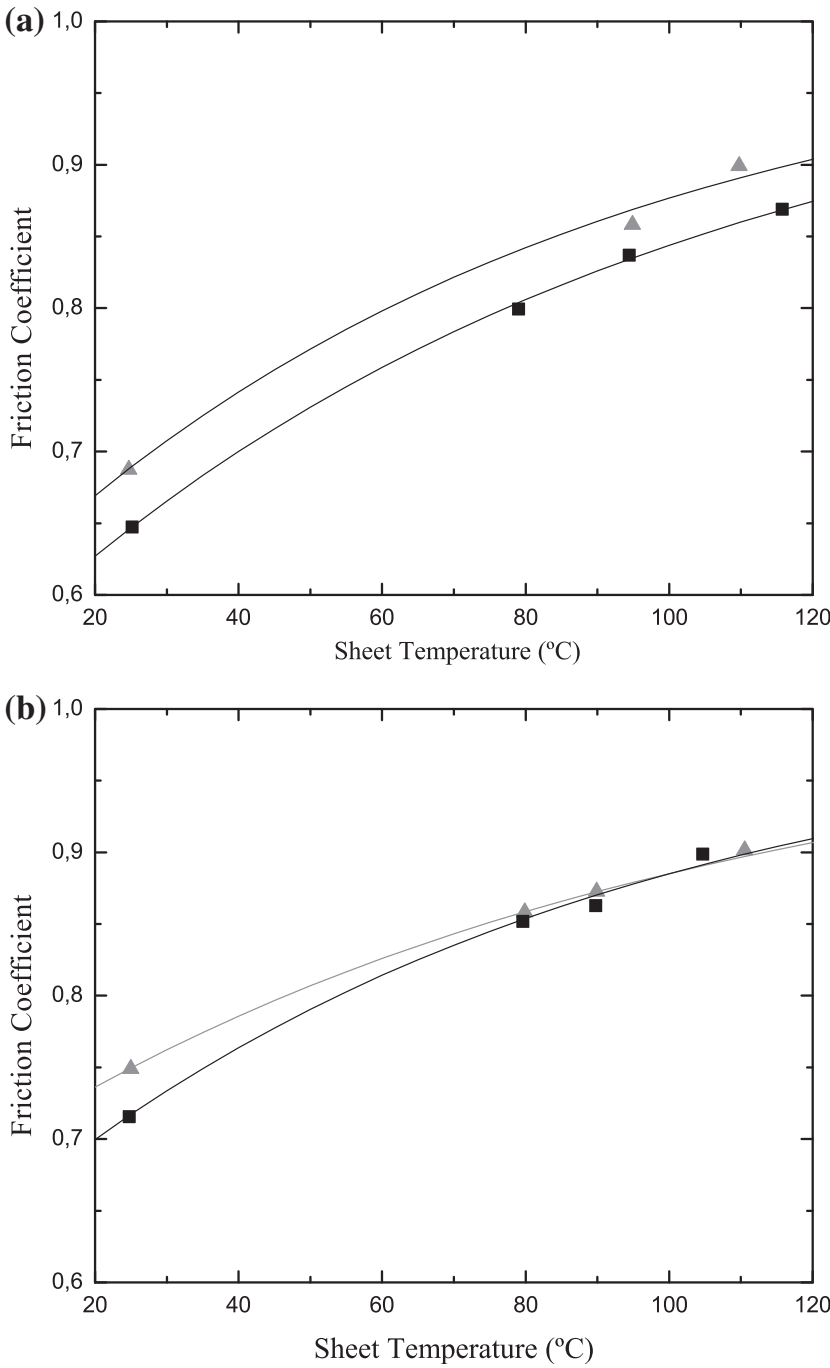

Fig. 4. Variation of the dynamic friction coefficient $\left(\mu_{d}\right)$ of the HIPS-steel and HIPSaluminum system according to the sheet temperature with an initial velocity of: (a) $1.6 \mathrm{~m} / \mathrm{s}$ and (b) $2.1 \mathrm{~m} / \mathrm{s}$.

more adhesiveness measurements than friction measurements shall be made, since there is no displace between the samples. For that reason, dynamic friction may not be considered.

\subsubsection{Effect of the mobile device displacement velocity}

Although some studies $[9,19]$ showed the influence of the penetration velocity of the plug in the plug-sheet $\mu_{d}$, it shall be considered that in the typical thermoforming processes, the range in which penetration velocities may vary is relatively small. Such velocities are produced by the plug displacement. This behavior may be verified by the measurements made to $\mu_{d}$ with initial different displacement velocities of the mobile device (Fig. 5).

It is shown how for values higher than $1.5 \mathrm{~m} / \mathrm{s}$ it was not observed significant $\mu_{d}$ variations because all the values are part of the experimental error. In Fig. 5, it is represented by a gray line the range in which all the values coincide. That fact may have a practical importance; since the variations that may occurred in the penetration velocity of the molding tools (molds and plugs) are very limited, the changes may have a less effect in the friction coefficient and may not caused alterations, among other characteristics, in the final piece thickness.

It is an interesting result due to the deformation velocity of the sheet alters the thickness distribution, because it is made of

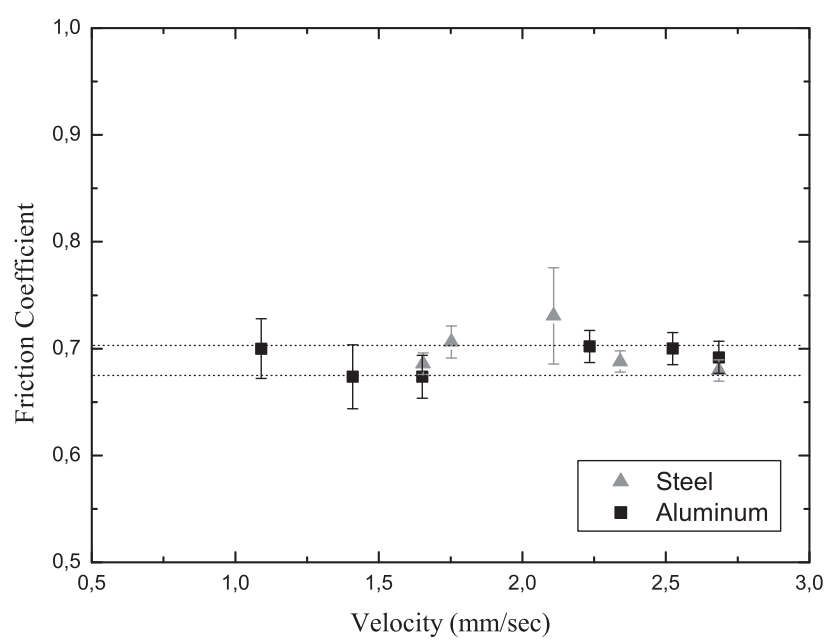

Fig. 5. Dynamic friction coefficient $\left(\mu_{d}\right)$ of the HIPS-aluminum and HIPS-steel system, considering the different displacement of the mobile device.

polymeric material and its behavior is viscoelastic. In other words, it may be one of the elastic solid ideal cases (in which a deformation depends on only in the applied force velocity) [22,23]. The viscous effect may be even higher at high temperatures. The fact that, in the variation interval of a thermoforming process, the friction coefficient keeps practically constant, entails that at the moment of modeling, the deformation and its behavior regarding the velocity shall be the one less variable to consider, which may considerably simplified the already very complex calculations.

\subsubsection{Plug materials}

As may be observed in Figs. 4 and 5, the aluminum and steel samples had a similar behavior when modifying the sheet displacement and temperature velocity, considering the experimental error. In other words, it was not possible detect significant $\mu_{d}$ variations regarding the plug material. The reason of this behavior may be the own nature of the effects that the materials have on the friction only when the interaction is on very polished surfaces. In this case, other types of interactions, like Van der Wall forces, may have important effects.

For experimental conditions used in this work, the roughness levels of the polymeric samples are higher for metal to achieve a substantial effect of the intermolecular forces. Here, it may be the roughness level that shall determine in which way the surfaces interact. Since they are samples with the same superficial finishing, it may be logic to think that $\mu_{d}$ values shall be similar, although made from different materials.

Nevertheless, in Fig. 6, it is represented how when modifying the nature of the polymeric material (in this case, a semi-crystalline resin such PP), there is a bigger difference between the use of steel or aluminum. Additionally, there are fewer $\mu_{d}$ values in the whole evaluated temperature range. Based on that, the finding differences when analyzing a material with semi-crystalline nature may be caused by the differences between the specific heats and thermal conductivity of the used materials. Such materials determined the amount of materials that shall be dragged by the plug and the way how the material is distributed. The appropriated selection of the plug material and its shape shall affect the thickness distribution of the assisted forming process. However, it has been reported by several authors that the heat transfer and the displacement phenomenon between the polymeric sheet and the plug are the variables that affect the most $[12,14,15]$. In addition, the surface roughness, the interface temperature, the contact pressure, and the penetration velocity are also affecting variables. 


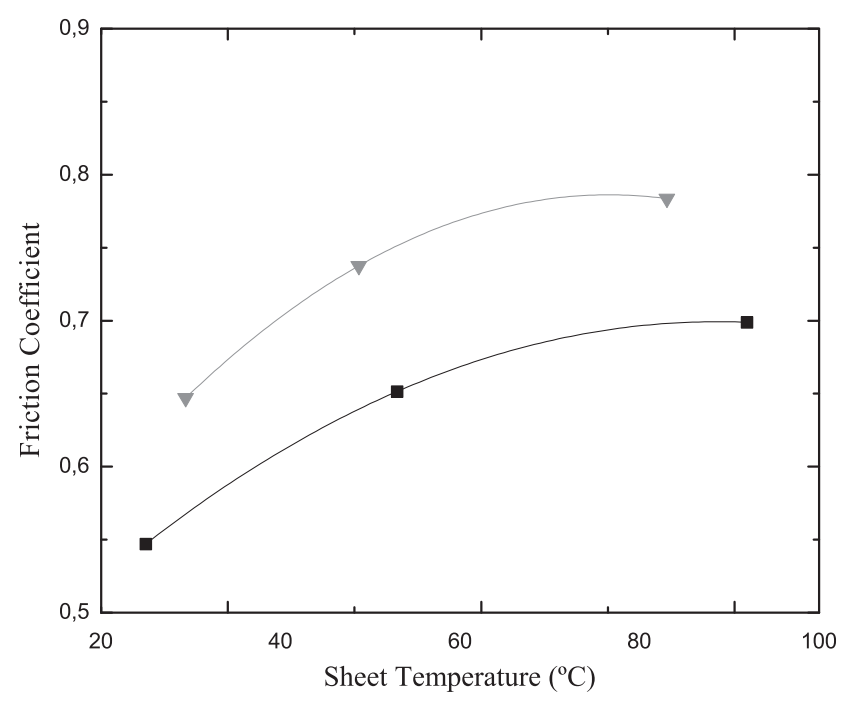

Fig. 6. Variation of the dynamic friction coefficient variation $\left(\mu_{d}\right)$ of the PP-steel and PP-aluminum system according to the sheet temperature with an initial velocity of $1.6 \mathrm{~m} / \mathrm{s}$.

\subsubsection{Superficial finishing}

Based on the aforementioned results, it was decided to modify the superficial finishing of the metallic surfaces with the help of a minor grain sanding. As above mentioned in Section 4, the HIPS-steel and HIPS-aluminum systems were sanded with a minor grain 75 GRIT sandpaper. Thus, there were evaluated the systems, which were sanding with a 360 GRIT sandpaper. Nevertheless, a roughness decrease did not cause variations in the $\mu_{d}$ values for the same aluminum-plastic and steel-plastic system, remaining the values around 0.70 . In this particular case, it may be possible that the reason of this behavior was caused by the high roughness of the HIPS sheet used in this study-enough high to cause scratches and visible erosion of the metallic material after several measurements $(>7)$. For this reason, it was necessary to finish the piece after each ten measurements.

Since it is an interfacial phenomenon, the friction is determined by properties of the two involved surfaces. In case that one the surfaces may have a big influence over the system behavior, big changes shall be required in the properties of the other surface to observe its changes. In this case, the superficial finishing that shall be obtained in the typical processes of sheet extrusion for thermoforming in relatively thick, with roughness from 0.8 to $12.5 \mu \mathrm{m}$ [24].

By other hand, the mechanical processes through which the molding pieces used in the thermoforming process, like the rectification and the turning, originated thinner superficial finishing: usually between 0.8 and $6.3 \mu \mathrm{m}$ for the turning, and even lower for the turning between 0.2 and $1.6 \mu \mathrm{m}$ [24]. The changes that maybe applied to the finishing inside these ranges shall not affect the frictional behavior, if the other element is a thick sheet like the one obtained by an extrusion process. Then, put it into practice, it is difficult to try to alter the friction coefficient of a system with alterations in the superficial finishing of the same system, because by one hand, it may be possible that for the size of the piece, there were only available a couple of techniques that make similar superficial finishing, and by other hand, the suffered erosion of the material continuous interaction with the plastic alters significantly its finishing.

\subsection{Validation}

Morales and Candal [21] in a previous study made a validation for a six cavities mold for HIPS glasses. When the thickness

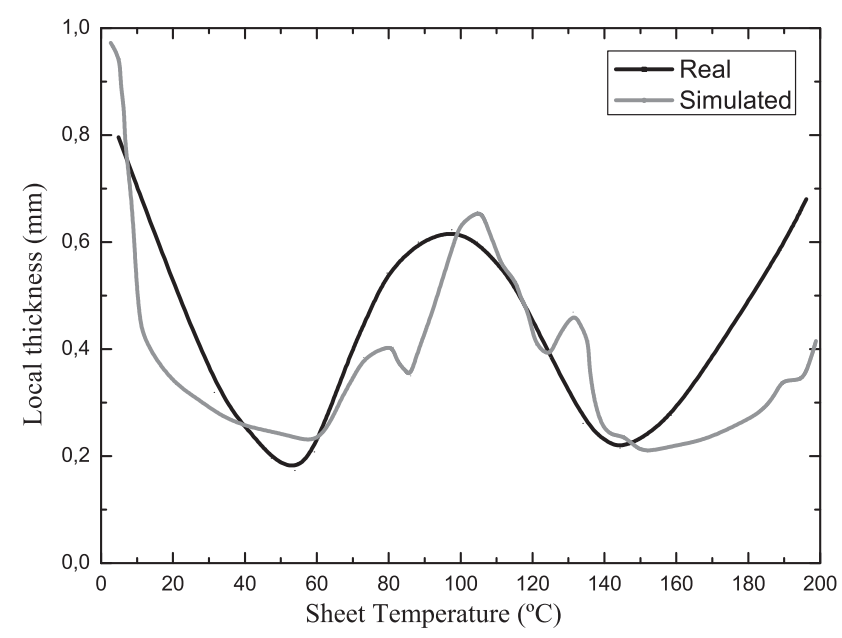

Fig. 7. Comparison between the thickness distribution of the estimated wall and the real ones for a thermoforming piece at $160{ }^{\circ} \mathrm{C}$ with a steel plug.

distribution of the thermoforming piece walls was compared to the ones simulated in a commercial program, the same tendency was observed. However, the thickness distribution average data reported by the program notably differ from the experimental, due to the considered approaches to make the simulations. One of these considerations was that for the simulations, it was considered a constant value of the friction coefficient recommended by the program for the HIPS sheet material/steel plug, without depending of neither the sheet temperature, nor penetration velocity of the plug. As it may be evidenced in this study, $\mu_{d}$ depend of these factors.

In this study, when it was made the validation of the mold used by Morales and Candal [21], there were not significant evidences in the distribution prediction of the thickness offered by the commercial program with the experimental, especially in the most critic zones (button and walls). In this case, it was considered that the $\mu_{d}$ determined by the suggested method (Fig. 7). The light variations may be caused by the erosion shown by the used plugs that tends to diminish the superficial sheet interaction. Moreover, it may be possible that the possible adherence sheet/plug by the high transfer heat transfer coefficient of the steel regarding HIPS. The fact that the simulated thickness variations are more "abrupt" than the real ones is due to the measurement distance in the real values (there were taken only five points along the cutting line). Additionally, it was made a simulation study considering not only the effect of the plug characteristics (material and form), but also its thermal properties [25].

\section{Conclusions}

Based on the methodology applied and the results obtained, it can be concluded that:

- The obtained real data of the frictional behavior (sheet-plug) shall allow achieving more precise simulations using data not assuming an arbitrary way. Having more reliable simulations, results may be used without coming after experimental variations that may waste time and money.

- If it is try to modify the frictional action between a plug or mold and polymeric sheet in real process conditions, the sheet temperature is the most incidental variable.

- Inside the process window of the material, it is possible to cause the adhesion of the sheet to the plug when the plug material is modified, and in such situation, the resistant of melt plays a bigger role that the friction when determining the thickness distribution. 
- The superficial finishing of the plug do not have a big effect in the frictional behavior, if the other element is a thick sheet like the one obtained by an extrusion process.

\section{References}

[1] Illig A. Thermoforming. A practical guide. Münich: Hanser Publisher; 2001.

[2] Throne JL. Understanding thermoforming. Münich: Hanser Publisher; 1999.

[3] Gruenwald G. Thermoforming. A plastic processing guide. Pennsylvania: Technomic Publishing; 1987.

[4] Hamada K, Okumura T, Ohno E, Yokoyama A. Optimum analysis method for thermoforming process by numerical approach. In: SPÉs ANTEC conference proceedings. Society of Plastic Engineers; 2001. w/p.

[5] Debergue P, Laroche D. Solid finite elements for the prediction of complex sheet distortions. In: SPÉs ANTEC conference proceedings. Society of Plastic Engineers; 2002. w/p.

[6] Sala G, Di Landro L, Cassago D. A numerical and experimental approach to optimise sheet stamping technologies: polymers thermoforming. Mater Des 2002;23(1):21-39.

[7] Cristopherson R, Debbaut B, Rubin Y. Simulation of pharmaceutical blister pack thermoforming using a non-isothermal integral model. In: SPÉs ANTEC conference proceedings. Society of Plastic Engineers; 2002. p. 773-7.

[8] Laroche D, Connolly R, Ellie C, Gundjian M. Thermoforming simulation and experimental validation on a bass boat hull. In: SPÉs ANTEC conference proceedings. Society of Plastic Engineers; 2000. w/p.

[9] Poller S, Michaeli W. Film temperatures determine the wall thickness of thermoformed parts. In: SPÉs ANTEC conference proceedings. Society of Plastic Engineers; 1992. p. 104-8.

[10] Gasco MC, Rodríguez F, Long T. Polymer-polymer friction as a function of test speed. J Appl Polym Sci 1998;67(11):1831-6.

[11] Martin NJ, Lappin JF, Harkin-Jones EMA, Martin PJ. The use of hot impact testing in the simulation of the plug-assisted thermoforming process. In: SPÉs ANTEC conference proceedings. Society of Plastic Engineers; 2000. w/p.

[12] Harron GW, Harkin-Jones EMA, Martin PJ. An experimental investigation of the plug assist thermoforming process. In: SPÉs ANTEC conference proceedings. Society of Plastic Engineers; 2001. w/p.
[13] Tulsian A, Mead J, Orroth S. Computer simulation of the effect of coefficient of friction in plug assist thermoforming. In: SPÉs ANTEC conference proceedings. Society of Plastic Engineers; 2004. p. 904-8.

[14] Collins P, Martin P, Harkin-Jones E, Laroche D. Experimental investigation of slip in plug-assisted thermoforming. In: SPÉs ANTEC conference proceedings. Society of Plastic Engineers; 2001. w/p.

[15] Laroche D, Collins P, Martin P. Modeling of the effect of slip in plug-assisted thermoforming. In: SPÉs ANTEC conference proceedings. Society of Plastic Engineers; 2001. w/p.

[16] Hegemann B, Eyere P. Polymer-Polymer friction at temperatures y rates simulating the thermoforming process. In: SPÉs ANTEC conference proceedings. Society of Plastic Engineers; 2003. p. 791-5.

[17] Sahin M, Çetinarslan CS, Erol H. Effect of surface roughness on friction coefficients during upsetting processes for different materials. Mater Des 2007;28(2):633-40.

[18] Sanchez-soto M, Martinez AB, Santana OO, Gordillo A. On the application of a damped model to the falling weight impact characterization of glass beadspolystyrene composites. J Appl Polym Sci 2004;93(3):1271-84.

[19] Martínez AB, Sánchez-soto M, Velasco JI, Maspoch MLl, Santana OO, Gordillo A Impact characterization of a carbon fiber-epoxy laminate using a nonconservative model. J Appl Polym Sci 2005;97(6):2256-63.

[20] Macaulay MA. Introduction to impact engineering. London: Chapman\&Hall; 1987. 201-30.

[21] Morales RA, Candal MV. Diseño y Fabricación de un Molde de Termoformado utilizando Herramientas CAD/CAE. Revista de la Facultad de Ingeniería de la UCV 2006;21(1):83-100.

[22] Novotný P, Sáha P, Kouba K. Fitting of K-BKZ model parameters for the simulation of thermoforming. Int Polym Proc 1999;3:291-5.

[23] Hegemann B, Liebing D, Eyerer P. Development of a measurement technique for tailored material characterization and validation of thermoforming simulation. In: SPÉs ANTEC conference proceedings. Society of Plastic Engineers; 2004. p. 909-13.

[24] González C, Zeleny R. Metrología Dimensional. Mexico: McGraw-Hill; 2000. p. $5-33$.

[25] Morales RA, Candal MV. Methodology for the determination of the biaxial measurements of plastic materials in a universal testing machine. In: SPÉs ANTEC conference proceedings. Society of Plastic Engineers; 2013. w/p. 